infection. We present 3 different courses of the HBV mother-to-child infections as a basis to differentiation of the therapeutic models

Methods We investigated case reports of 3 children infected with $\mathrm{HBV}$ by their mothers $\mathrm{HBsAg}(+) \mathrm{HBeAg}(+)$. Chronic hepatitis B was confirmed in mothers aged 18, 21 and 26 respectively. All children were vaccinated against hepatitis B at delivery: two of them three times, one two times. One of the children was administered HBIG in the first day of its life.

Results Hepatitis B virus infection in 2 children was revealed in the 3rd year of life. Acute hepatitis with the Gianotti-Crosti syndrome was diagnosed in 1 child in the $6^{\text {th }}$ month of life. Subsequently, all children were diagnosed with chronic hepatitis B and the course of the disease was different in each case. In the first child aged 1, the activity of alanine aminotransferase decreased to near normal level with the seroconversion of $\mathrm{HBe}$ antigen to antibodies anti-HBe. The second child in the fourth year of life has high level of $\mathrm{HBV}$ viral load and high activity of alanine aminotransferase. The third child (12 years old) has exacerbation of disease after failure of treatment (lamivudine, interferon twice).

Conclusions 1 . The course of chronic hepatitis B in children after maternal infection may be vary, therefore some adjustments in treatment should be taken into account.

\section{INFECTIOUS ERYTHEMA NODOSUM}

doi:10.1136/archdischild-2012-302724.0885

${ }^{1} \mathrm{H}$ Rahmoune, 'N Boutrid, ${ }^{2} \mathrm{H}$ Torki, ${ }^{3} \mathrm{~S}$ Chehad, 'B Bioud. 'Pediatrics, University Hospital of Setif; ${ }^{2}$ Pediatrics, EHS Mère-Enfant, El-Eulma, Setif; ${ }^{3}$ Dermatology, University Hospital of Constantine, Constantine, Algeria

Introduction Erythema nodosum (EN) is a dermatological entity can belong to several causes. We describe two cases, side two of the offending pathogens.

Material and Method Case 1: A little boy of 7 months was admitted for febrile erythema nodosum

The history, by cons, reveals a close tuberculosis contact:the father was treated for pulmonary tuberculosis, but no chemoprophylaxis has been lavished on the family.

High inflammatory markers and a $14 \mathrm{~mm}$-tuberculin test are holding a post-tuberculosis EN. Antibiotic treatment allows bioclinical resolution.

Case 2: A 5 year old girl was admitted for acute EN. She has, outside of a purulent amygdalytis, no other pathological signs.

In addition to high ESR and CRP, the results found for ASLO = 800 ui.

The rapid resolution in antibiotic anti-streptococcal etiology confirms the suspicion.

Results and discussion: The EN is the most common inflammatory nodules or panniculitis.

Investigation of an $\mathrm{EN}$ is often much custom and takes particular account of local epidemiology, history, geographic origin and associated signs evoking a particular pathology.

Discussion of these cases can raise some discussion points:

- The place still occupied worrying Mycobacterium tuberculosis in pediatric morbidity

- B-hemolytic streptococcus is a public health problem

The value of prevention, secondary and tertiary, deserves an ongoing effort on targeted risk populations.

Conclusion The EN is dogmatically infectious first.

Streptococcal infection is currently the most common cause, after eliminating a primary tuberculosis. PARAPNEUMONIC PLEURISY

doi:10.1136/archdischild-2012-302724.0886
S Diaconescu, C Olaru, G Paduraru, M Burlea. 'Gr.T.Popa' University of Medecine and Pharmacy, lasi, Romania

Background and Aims Chickenpox is an acute, benign, highly contagious disease characterized by generalized vesicular exanthema with self-limited evolution. Pneumonia is the most serious complication of varicella, occurring more frequently in adults $(>20 \%)$ than in children. An outbreak of the disease started in late autumn 2011 in Romania and continues in present.

Methods and results: The authors present the case of a 4 years old boy admitted into the Infectious Diseases Hospital with chickenpox. On the 4-th day of the disease high fever, dyspnea with tachypnea, intercostal retractions, pleuritic pain and cough appeared and the patient was transferred to the Children Hospital. Clinical examination showed abolished left basal vesicular breath sound with wet crackles in the middle and superior lung area; chest X-ray finded inferior left lobe pneumonia and mild pleural effusion. Tracheal aspirate culture was negative. Leucokytosis with neutrophilia and increased ESR and C-reactive protein was founded. Broad spectrum antibiotherapy was started with favourable evolution after 3 weeks.

Conclusions Among the most serious complications of varicella is pneumonia; it is less common in children than in adults but it may lead to death. However, the epidemic status in Romania in 2011-2012 was associated with an increased number of viral pneumonias; in our case the radiological aspect was highly suggestive for a secondary bacterial infection even with negative aspirate culture (explained by prior antibiotherapy). The history for chickenpox vaccine was negative in our patient. In Romania, the chickenpox immunization is not included into the National Programe of Immunisations at this moment.

\section{TWO CASES OF GIGANTIC JUVENILE CYSTIC ECHINOCOCCOSIS}

doi:10.1136/archdischild-2012-302724.0887

${ }^{1} \mathrm{~K}$ grosse Kreymborg, ${ }^{2} \mathrm{M}$ Stojkovic, ${ }^{2} \mathrm{~T}$ Junghanss, ${ }^{3} \mathrm{M}$ Hirschburger, ${ }^{1} \mathrm{KP}$ Zimmer. ${ }^{1}$ Department of General Pediatrics and Neonatology, University of Giessen, Giessen; ${ }^{2}$ Section Clinical Tropical Medicine, Heidelberg University Hospital, Heidelberg; ${ }^{3}$ Department of General and Thoracic Surgery, University of Giessen, Giessen, Germany

Background and Aims Cystic Echinococcosis (CE) is a serious multi-organ disease, caused by cestode infection with Echinococcus granulosus. Simultaneous hepatopulmonary or isolated pulmonary hydatidosis in children are rare and demand an individual, but often multidisciplinary case management.

Methods We report on two gigantic CE-manifestations in children. The first case was a 4-year-old boy, presenting with severe pneumonia and abdominal pain in case of hepatopulmonary hydatidosis. The second case was a 6-year-old boy, who presented with continuous coughing in case of isolated, bilateral pulmonary hydatidosis. While the 4-year-old displayed a severely reduced state of health, the 6-year-old showed good general condition.

Results Serologic tests for Echinococcus granulosus infection were negative in either case. The diagnosis of CE was solely based on diverse imaging methods in both entities. While the 4-year-old boy was first treated for his secondary pneumonia, the 6-year-old demanded imminent anthelmintic and surgical treatment due to a ruptured pulmonary cyst with threat of secondary agent dissemination. Finally both patients were discharged after a two-step surgical cyst removal and with continued anthelmintic longterm therapy, which led to restitutio ad integrum in either case.

Conclusions Although a proper multidisciplinary CE-management has evolved in the past decades, an evidence-based evaluation of its outcome, especially in children, is not yet available. Serologic tests for CE-infection are very often tested false-negative, so that the initial diagnosis is mainly image-based. The urge of anthelmintic 
and/or surgical treatment depends on the Stage of the CE-infection, not on the general condition of the patient.

\section{ANTIBIOTICS STUDY ON STAPHYLOCOCCUS SPP. STRAINS ISOLATED FROM VENOUS AND URINARY CATHETERS IN NICU OF HAMADAN HOSPITALS, WEST RAN}

doi:10.1136/archdischild-2012-302724.0888

'R Yousefimashouf, ${ }^{2 N}$ Molazadeh. 'Department of Microbiology, Research Center for Molecular Medicine of Hamadan University of Medical Sciences; ${ }^{2}$ Faculty of Medicine, Hamadan University of Medical Sciences, Hamadan, Iran

Background and Aim Staphylococcus coagulase negative strains are colonized on epiderm and distribute in environment and outer bodies apparatus such as protez and intera-venous catheters. The aim of this study was the frequency of Staphylococcus coagulase negative isolated from venous and catheters children hospitalized in NICU of Hamadan hospitals and determination of antibiotics resistance patterns in Hamadan, the west of Iran

Methods We collected 108 samples randomly from patients who were hospitalized in NICU hospitals of Hamadan and they needed to venous or urinary catheters. One specimen of each patient was taken and inoculated into carrier transported media and transferred to bacteriology laboratory to identification of strains. Antibiogram was performed by Kirby-Bauer method. Data was analyzed using SPSS 15 software.

Results Out of 108 tested samples, $32.7 \%$ of patients had urinary catheter and $67.3 \%$ had venous catheter. $28 \%$ of tested samples had positive culture. The positive cases were significantly found in those children who had been used catheter more than 48 hours $(P=0.00)$. From the positive cases, Staphylococcus epidemidis (40.4\%), Acinetobacter baumannii (10.6\%) and E. coli (8.5\%) were the most common isolates. The most rate of resistance of Staphylococcus epidemidis was against to erythromycin and ampicillin. The most rate of sensitivty of E. coli was against to gentamicin and Psuedomonas aeruginosa to ciprofloxacin.

Conclusion Our results showed the high contamination in used catheters particularly in those patients who needed to catheter for long time. We also indicated the high drug resistance in strains isolated from catheters.

\section{ETIOLOGIES OF THE STATUS EPILEPTICUS IN CHILDREN HOSPITALISED IN THE PICU OF THE UNIVERSITY HOSPITAL CENTER OF ORAN (ALGERIA)}

doi:10.1136/archdischild-2012-302724.0889

D Boumendil, MA Negadi, K El Halimi, H Bouguetof, ZC Mentouri. Pediatric Intensive Care Unit, Faculty of Medicine - Oran University, Oran, Algeria

Background and Aims Status Epilepticus is one of the most frequent neurological emergencies in Pediatrics, that can involve the vital and functional prognosis in the short and long term.

The Aim of this study is to determine epidemiological, causative, diagnostic aspects and to evaluate the therapeutic means.

Methods In this prospective study we analyse 214 children with status epilepticus between January 2008 and December 2010.

Results The mean age is 04 years (min: 28 days - max: 15 years) with a sex ratio equal to 1.3 . $60 \%$ of cases was febrile. $81 \%$ of the seizures are generalized. The differents etiologies are: Epilepsy: 59 (27.5\%); Occasional seizures: 69 (32.24\%); Infections of central nervous system (CNS): 53 (24.7\%) (26 méningitidis and 27 méningoencéphalitis); Febrile seizures: 24 (11\%); Indeterminate cause: 09 (04\%).

It was noted a long delay between the onset of clinical manifestations and world-renowned workplace support. The drugs used are represented by injectable diazepam and phenobarbital. In terms of support, $46 \%$ need artificial ventilation. In this series the mortality is $22 \%$.

Conclusion The vital and functional prognosis could be improved by better prevention and effective treatment of infectious diseases, a reduction of the period of support and better ways of resuscitation

\section{URINARY TRACT INFECTION AND PREDISPOSING FACTORS IN CHILDREN}

doi:10.1136/archdischild-2012-302724.0890

'M Naseri, ${ }^{2}$ SA Alamdaran. ${ }^{1}$ Pediatric Nephrology; ${ }^{2}$ Radiology, Mashhad University of Medical Sciences, Mashhad, Iran

Objective This study was designed to determine the predisposing factors in children with symptomatic urinary tract infection (UTI) according to age and gender.

Material and Methods We reviewed prospectively 183 pediatric patients with symptomatic UTI admitted to emergency department or referred to nephrology clinic from November 2002 through July 2005. All patients underwent renal ultrasonography and voiding-cystouretherography or radionuclide cystography. Diuretic renal scan or intravenous pyelography (IVP) was performed in those with urinary system dilatation. Urodynamic studies were done in patients with normal radiologic findings and recurrent infections or urinary-intestinal symptoms.

Findings Of 183 patients, 130 cases (71\%) were female and 53 patients (29\%) male. Most of the patients (61.9\%) were between 2-24 months old ( $\mathrm{P}=0 / 001)$. Vesicoureteral reflux (VUR) was the most common predisposing factor in both genders $(46.9 \%$ in girls and $48.9 \%$ in boys). Voiding dysfunction in girls and urinary obstruction in boys were found with a significant difference $(\mathrm{P}=0 / 03$ for both). In all age groups, except patients $\leq 1$ month, the most common predisposing factor was reflux. Reflux, urinary obstruction and nephrolithiasis were found with a significant difference in 2-24 months age group ( $\mathrm{P}=0 / 001$ for all).

Conclusion In our study vesicoureteral reflux (VUR) was as common in boys as in girls, and suggested urolithiasis as a significant UTI predisposing factor. This study showed that voiding dysfunction in girls and urinary obstruction in boys are as significant predisposing factors. We suggest urodynamic studies in patients with normal radiologic findings and recurrent infections or urinaryintestinal symptoms

\section{ACUTE PYELONEPHRITIS AND DIAGNOSTIC PARAMETERS}

doi:10.1136/archdischild-2012-302724.0891

'VN Stavileci, 'M Begolli, ${ }^{2} \mathrm{~A}$ Keka. ${ }^{1}$ Nephrology, University Clinical Centre of Kosova, Pediatric Clinic; ${ }^{2}$ University Clinical Centre of Kosova, Prishtina, Kosovo

Introduction Diagnosis and the right time management of Pyelonephritis are extremely important especially if we consider the risk of permanent kidney damage.

Objectives and research To analyze the clinical and laboratory signs and radiological presentation of the disease in children diagnosed with acute pyelonephritits during year 2010 in Pediatric clinic, Nephrology Unit.

Methods Among cases admitted to Nephrology Unit diagnosed as pyelonephritis acuta, during 2010, we analyzed presentation symptoms by age, inflammatory laboratory results, protein degradation products, urine and kidney ultrasound findings.

Results Among of 83 cases with urinary tract infections, 32.5\% were diagnosed as pyelonephritis acuta. More frequent among male infants and preschool age and on female school age. $29.6 \%$ of the cases were male and $70.3 \%$ of the cases were female. Inflammatory parameters were high in $88.8 \%$ of cases and the value of above SE: $100 \mathrm{~mm} / \mathrm{h}$ was in $20.8 \%$ of cases. Dominated presentation 